\title{
Single Embryo Transfer in IVF to Prevent Multiple Pregnancies
}

\author{
James Catt, Tina Wood, Michael Henman, and Robert Jansen \\ Sydney IVF, Australia
}

\begin{abstract}
$\Lambda \mathrm{s}$ the success rates of IVF clinics improve, one of the adverse consequences is the increased incidence of twins, due largely to the number of embryos transferred. Even if the number of embryos transferred is restricted to two, the twinning rate can exceed $40 \%$ of the pregnancies. An obvious way to reduce this high twin rate would be to transfer only one embryo. This would require that cryopreservation of the supernumerary embryos be efficacious enough so that the chance of achieving an ongoing pregnancy is not diminished by transferring a single embryo in the stimulated cycle. Previous studies utilising embryos on day 2 and 3 of development have shown that the pregnancy rates can be acceptable (about $40 \%$ ) and that the cumulative rate can be up to $60 \%$. Most of these studies, however, do not include a comparison with the cumulative pregnancy rate with two embryos transferred in the stimulated cycle. Therefore, the efficacy has not been proven. We present clinical data from the past few years to illustrate the increase in success rates and the concomitant increase in twinning rates. The increased success in the cryopreservation program has enabled us to trial a single embryo transfer program and compare the results to the transfer of two embryos. The results strongly suggest that the transfer of a single embryo is the better clinical option.
\end{abstract}

Multiple pregnancies, including twin pregnancies, are well known to have many adverse effects both on fetal and maternal health and long-term effects on both the parents and offspring (Elster, 2000; Olivennes, 2000).

Subfertile and infertile couples often present at IVF clinics after several years of attempting to have a baby without success. IVF over the past couple of decades has conventionally transferred a minimum of two embryos to attempt to increase the chances of a successful pregnancy. The freezing of supernumerary embryos has become commonplace in most clinics and the pregnancy rates, although variable, have been acceptable. However, as culture conditions and patient care has improved, so have success rates. This has led to an increase in twinning rates even with transferring only two embryos (Gerris \& Van Royen, 2000). A recent study in Australia by Umstad and Gronow (2003) showed that IVF clinics were responsible for an increasing percentage of twin deliveries and hence putting a strain on health care facilities.

A number of questions need to be resolved to ensure that optimal results are being achieved without compromising the patient or their potential offspring. Patients presenting for IVF treatment have to be educated and informed that having twins is not a desirable outcome and should be avoided if possible. One of the ways to convince patients to accept a single embryo transfer is to demonstrate that the freezing program is efficacious and that embryos can safely be frozen, thawed and give rise to pregnancies. Therefore there is a need to assess cumulative pregnancy rates for a single stimulated cycle. There is only one report, to date, that compares the cumulative pregnancy rates resulting from a single or two embryo transfer (Martikainen et al., 2001). The issue of cryopreservation is paramount since a good cryopreservation program will reduce the need to choose the best embryo since it will eventually be transferred after freezing. This does not mean, of course, that the quality of the transferred embryo is immaterial as the pregnancy rate from the fresh transfer is still important. Most studies, to date on single embryo transfer have concentrated on morphological criteria for selection (ESHRE, 2001; Gerris et al., 1999; Martikainen et al., 2001; Tiitinen et al., 2001; Vilska et al., 1999). Gardener et al. (2000) suggested growing embryos for another 2 days in culture as this would exert more of a selection pressure for the 'good' embryos than 3 days in culture. We report here the clinical results from our unit and follow the evolution of the program to accommodate the increasing success rates with a concomitant increase in twinning.

\section{Materials and Methods}

Clinical data from 1998 to 2001 was used to assess the pregnancy and twin pregnancy rates in patients aged less than 38. All patients had long down regulation and direct ovarian stimulation using FSH. Oocytes were retrieved by ultrasound guided follicle aspiration and oocytes inseminated by either conventional IVF or ICSI according to the sperm parameters. All zygotes produced were grown for 5 days post-insemination using stage specific media (SIVF media). Suitable supernumerary embryos were frozen for transfer in subsequent frozen embryo cycles.

Eleven days after embryo transfer a quantitative $\beta$-hCG was performed and a positive pregnancy test was recorded if the level was greater than $50 \mathrm{IU} / \mathrm{ml}$. A viable pregnancy was determined 2-3 weeks later by a demonstrable fetal heart on ultrasound. This was defined as a clinical pregnancy and two fetal hearts defined as a twin pregnancy.

Address for correspondence: James Catt, Sydney IVF, GPO Box 4384 NSW 2001, Australia. Email: jim.catt@sivf.com.au 
For the elective Single Embryo Transfer (eSET) trial, patients were invited to have a single embryo transferred if they were aged less than 38 , had at least two embryos suitable for transfer and had less than three previous unsuccessful IVF cycles. The patients declining a single embryo transfer had two embryos transferred and served as the control group. Patients not achieving a clinical pregnancy usually returned for a frozen embryo transfer cycle and the number of embryos transferred was a maximum of two but unrelated to the number transferred in the fresh cycle.

A cumulative fetal heart pregnancy rate was calculated per stimulated cycle and the percentage of patients that had a clinical pregnancy calculated along with twinning data.

\section{$\overline{\text { Results }}$}

Figure 1 shows the clinical pregnancy rate over time at Sydney IVF. Since 1998 there has been a steady increase in this rate from $28 \%$ in 1998 to $52 \%$ in 2001 . The corre-

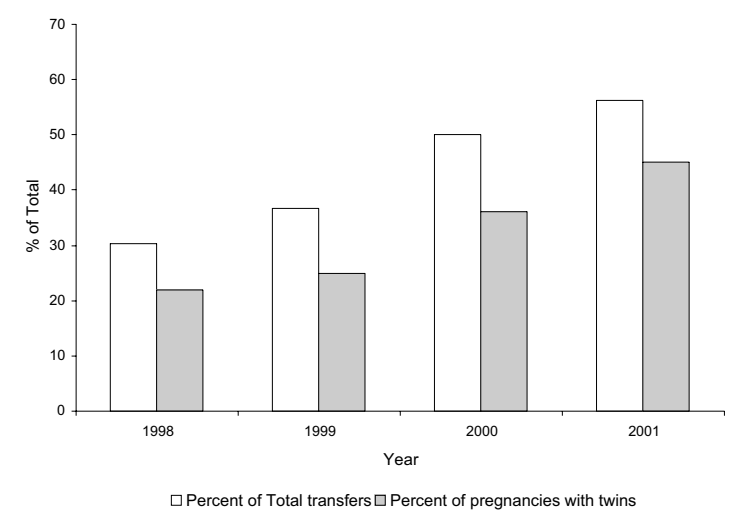

Figure 1

Bar chart showing the percent of embryo transfers resulting in a viable pregnancy between 1998 and 2001 for patients aged below 38. sponding twinning rates were $20 \%$ and $38 \%$ respectively. As the clinic has become bigger so the patient number has increased (approximately doubled) so that the total number of twin pregnancies has also risen dramatically from 32 in 1997 to 129 in 2001.

One of the prerequisites for moving to a single embryo transfer is the establishment of an efficacious cryopreservation program. Figure 2 shows an increase in implantation rates with time. It is noteworthy that the denominator used for the derived figures is on an embryo thawed basis not the usual per embryo transferred basis. The reason for this is that the embryos were frozen on the expectation that they would have been transferred if fresh and therefore the use of this denominator gives a more accurate picture of the status of the freezing program. The proportion of pregnancies that were twins, while not trivial at approximately $12 \%$ of pregnancies, is much less of a problem than the fresh transfers.

On the basis of increasing twin pregnancies and a growing confidence in the freezing program a single embryo transfer trial was initiated during 2001. Table 1 shows the clinical pregnancy rates from this study. It can immediately be seen that the clinical pregnancy rates for the two embryo transfer group are higher than for the single embryo transfer group, along with a concomitant high twinning rate, as expected. The pregnancy rate for single embryo transfers is still at an acceptable rate of over $40 \%$. There was one incidence of a monozygotic twin in the single embryo transfer group. As expected, there was no difference in the implantation rate per embryo transferred, as one would expect every embryo transferred to have an equal chance of implantation if they are of a suitable grade.

Table 2 shows the frozen embryo transfer cycles derived from the stimulation cycles given in Table 1 . In addition, the cumulative clinical pregnancy rate for each stimulated cycle is given. It can be seen from the data that frozen embryo cycles have added a number of clinical pregnancies to both groups, but more to the single embryo transfer

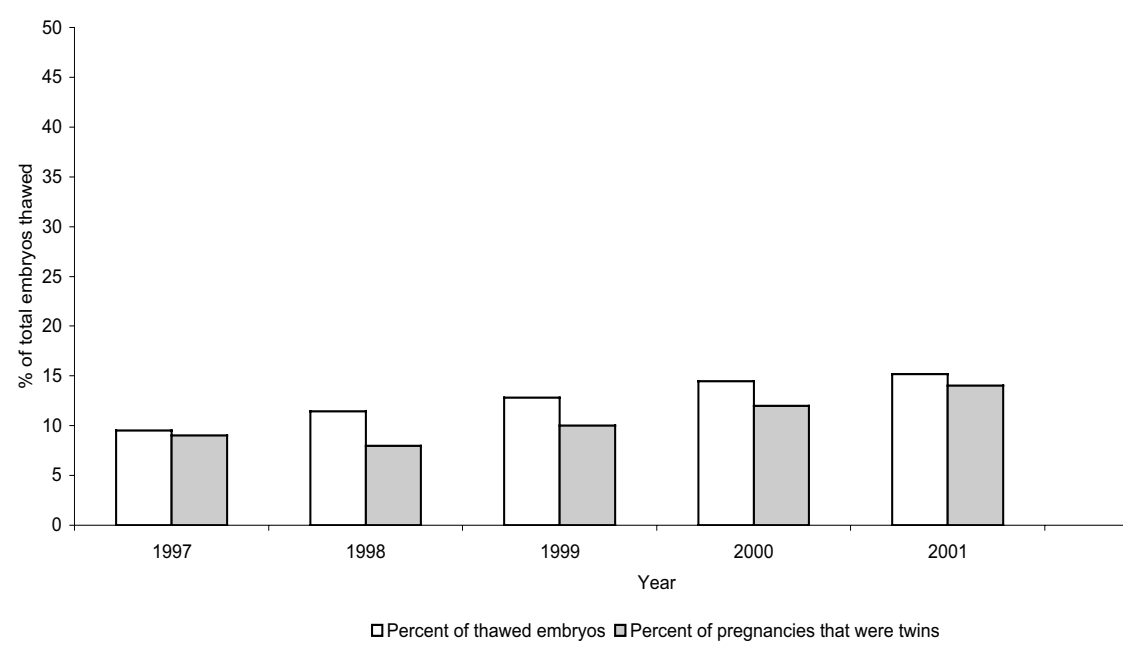

Figure 2

Bar chart showing the implantations per embryo thawed for embryos transferred in frozen embryo cycles between 1997 and 2001. 


\section{Table 1}

Pregnancies Resulting from the Transfer of Either One or Two Fresh Embryos

\begin{tabular}{lrr}
\hline & Single ET & $2 \times$ ET \\
\hline No. of embryo transfer procedures & 111 & 274 \\
No. clinical pregnancies & $49(44 \%)$ & $161(59 \%)$ \\
No. fetal hearts & 50 & 232 \\
(\% per embryo transferred) & $(45 \%)$ & $(42 \%)$ \\
No. twin pregnancies & 1 & 71 \\
(\% per pregnancy) & $(2 \%)$ & $(44 \%)$ \\
\hline
\end{tabular}

Table 2

Clinical Pregnancy Rate from Frozen Embryos Derived from Cycles with One or Two Embryos Transferred in the Stimulated Cycle

\begin{tabular}{lcc}
\hline & \multicolumn{3}{c}{ Embryo derived from } \\
& Single ET & 2 x ET \\
\hline No. frozen embryo transfers & 112 & 105 \\
No. clinical pregnancies (\% per ET) & $33(29 \%)$ & $29(28 \%)$ \\
No. fetal hearts (per embryo thawed) & $36(14 \%)$ & $32(12 \%)$ \\
Total no. of patients pregnant & 83 & 190 \\
$\%$ of patients pregnant & $74 \%$ & $70 \%$ \\
Overall twinning rate & $5 \%$ & $28 \%$ \\
\hline Note: The Cumulative Pregnancy Rates and Overall Twinning Rates Are also Shown
\end{tabular}

group. This would be expected if the freezing regime does not substantially harm the embryos. The table clearly shows that the cumulative pregnancy rates for both procedures results in the same proportion of patients becoming pregnant but with a vastly reduced twinning rate in the group that had a single embryo transferred in the fresh cycle (from nearly $30 \%$ of all pregnancies to $5 \%$ ).

\section{Discussion}

Multiple and, in particular, twin pregnancies arising from IVF, has been an emerging problem for several years now. Earlier work (Giorgetti et al., 1995; Hunalt et al., 2002; Van Royen et al., 1999;) focused on models based on embryo characteristics to predict patients potentially at risk of conceiving twins. While these can be of use they are not always accurate or are rather unwieldy to implement in a clinical setting. Furthermore, all of the previous work has been conducted on embryos either day 2 or 3 post-insemination. Gardener and Lane (1997) proposed that culturing embryos to day 5 post-insemination might allow a better discrimination between embryos. The study presented here used exclusively day 5 embryos and clearly demonstrates that acceptable pregnancy rates can be achieved using single embryo transfers in a relatively unselected population of IVF patients. This study confirms those conducted previously (Martikainen et al., 2001; Salumets et al., 2003; Tiitinen et al., 2001; Vilska et al., 1999) using day 3 embryos. Only one study (Tiitinen et al., 2001) has included cumulative pregnancy rate but unfortunately the data did not include the cumulative rate for those patients having two embryos transferred in their stimulated cycle. Therefore, while the cumulative pregnancy rate was acceptable, the key question as to whether the same proportion of patients became pregnant was not answered. In the current study we have followed both groups and found the same proportion of patients become pregnant while reducing the overall twinning rate at least fivefold.

Since the results of this study were known, single embryo transfer has been encouraged in clinical practice and over $60 \%$ of patients less than 38 years of age now have a single embryo transferred. Twins are, however, not eliminated because patients are not restricted to having a single embryo transfer in any subsequent cycles. The future challenge is now to decrease further the twinning rate in frozen embryo cycles and in older patients by identifying those at risk of conceiving twins.

\section{References}

Elster, N. (2000). Less is more: The risks of multiple births. Fertility and Sterility, 74, 617-623.

ESHRE Campus Course Report (2001). Prevention of twin pregnancies after IVF/ICSI by single embryo transfer. Human Reproduction, 16, 790-800.

Gerris, J., De Neubourg, D., Mangelschots, K., Van Royen, E., Van de Meersche, M., \& Valkenburg, M. (1999). Prevention of twin pregnancy after in vitro fertilisation or intracytoplasmic sperm injection based on strict embryo criteria: A prospective randomised clinical trial. Human Reproduction, 14, 2581-2587.

Gardener, D. K., \& Lane, M. (1997). Culture and selection of viable blastocysts: A feasible proposition for human IVF. Human Reproduction Update, 3, 367-382.

Gardener, D., Lane, M., Stevens, J., Schenkler, T., \& Schoolcraft, W. B. (2000). Blastocyst score effects implantation and pregnancy outcome: Towards a single blastocyst transfer. Fertility and Sterility, 73, 1155-1158.

Gerris, J., \& Van Royen, E. (2000). Avoiding multiple pregnancies in ART. A plea for single embryo transfer. Human Reproduction, 15, 1884-1888.

Giorgetti, C., Terriou, P., Auquier, P., Hans, E., Spach, J. L., Salzmann, J., \& Roulier, R. (1995). Embryo score to predict implantation after in vitro fertilization: Based on 957 single embryo transfers. Human Reproduction, 10, 2427-2431.

Hunault, C. C., Eijkemans, M. J. C., Pieters, M. H. E. C., te Velde, E. R., Habbema, J. D. F., Fauser, B. C. J. M., et al. (2002). A prediction model for selecting patients undergoing in vitro fertilization for elective single embryo transfer. Fertility and Sterility, 77, 725-732.

Martikainen, H., Tiitinen, A., Tomas, C., Tapanainen, J., Orava, M., Toumivaara, L., et al. (2001). One versus two embryo transfer after IVF and ICSI: A randomized study. Human Reproduction, 16, 1900-1903.

Olivennes, F. (2000). Double trouble: Yes a twin pregnancy is an adverse outcome. Human Reproduction, 15, 1663-1665.

Salumets, A., Hyden-Granskog, C., Makinen, S., Suikkari, A-M., Tiitinen, A., \& Tuuri, T. (2003). Early cleavage predicts the viability of human embryos in elective single embryo transfer procedures. Human Reproduction, 18, 821-825. 
Tiitinen, A., Halttunen, M., Harkki, P., Vouristo, P., \& HydenGranskog, C. (2001). Elective single embryo transfer: The value of cryopreservation. Human Reproduction, 16, $1140-1144$.

Umstad, M. P., \& Gronow, M. J. (2003). Multiple pregnancy: A modern epidemic? Medical Journal of Australia, 178, 613-615.
Van Royen, E., Mangelschots, K., De Neubourg, D., Valkenburg, M., Van de Meerssche, M., Ryckaert, G., et al. (1999). Characterization of a top quality embryo, a step towards single-embryo transfer. Human Reproduction, 14, 2345-2349.

Vilska, S., Tiitinen, A., Hyden-Granskog, C., \& Hovatta, O. (1999). Elective transfer of one embryo results in an acceptable pregnancy rate and eliminates the risk of multiple birth. Human Reproduction, 14, 2392-2395. 